\title{
Port Acadie
}

Revue interdisciplinaire en études acadiennes

An Interdisciplinary Review in Acadian Studies

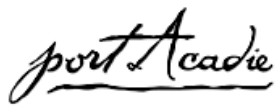

\section{Migrations des peuples africains en Europe et transferts des habitudes culturelles : le cas des Burkinabè de France}

\section{Patrice Kouraogo}

Numéro 13-14-15, printemps-automne 2008, printemps 2009

La résistance des marges : exploration, transfert et revitalisation des traditions populaires des francophonies d'Europe et d'Amérique

URI : https://id.erudit.org/iderudit/038436ar

DOI : https://doi.org/10.7202/038436ar

Aller au sommaire du numéro

Éditeur(s)

Université Sainte-Anne

ISSN

1498-7651 (imprimé)

1916-7334 (numérique)

Découvrir la revue

Citer cet article

Kouraogo, P. (2008). Migrations des peuples africains en Europe et transferts des habitudes culturelles : le cas des Burkinabè de France. Port Acadie, (13-14-15), 307-320. https://doi.org/10.7202/038436ar

\section{Résumé de l'article}

La migration, vécue dans le contexte africain en deux temps (pendant et après la colonisation) et en deux modes (contraignant et volontaire), est un vecteur privilégié de transferts culturels. Quand un groupe d'individus se déplace, c'est un échantillon représentatif d'une nation qui se déporte d'un espace à un autre. Il y a forcément une reproduction sociale et culturelle qui est assurée dans ce nouvel espace par la poignée de personnes déplacées, aussi petite soit-elle. Autant il y a eu migrations et transferts culturels de l'Europe à l'Amérique, autant on peut observer ce même phénomène du côté de l'Afrique vers l'Europe. Le Burkina-Faso, ancienne colonie française, compte plusieurs milliers de ses fils dans l'Hexagone. Ces derniers ont emporté avec eux des traditions, us et coutumes de leur pays. Consciemment ou inconsciemment, en terrain favorable ou hostile, ils reproduisent ces pratiques. Parmi elles, on peut citer la solidarité, la polygamie, le partage, le regroupement... Dans ce thème, nous allons démontrer que ce qui est valable en Amérique pour l'Europe l'est en Europe pour l'Afrique à travers l'exemple des Burkinabè vivant en France.
Ce document est protégé par la loi sur le droit d'auteur. L'utilisation des services d'Érudit (y compris la reproduction) est assujettie à sa politique d'utilisation que vous pouvez consulter en ligne.

https://apropos.erudit.org/fr/usagers/politique-dutilisation/ 


\title{
Migrations des peuples africains en Europe et transferts des habitudes culturelles : le cas des Burkinabè de France
}

\author{
Patrice Kouraogo \\ Ministère de la Culture du \\ Burkina-Faso
}

\begin{abstract}
Résumé
La migration, vécue dans le contexte africain en deux temps (pendant et après la colonisation) et en deux modes (contraignant et volontaire), est un vecteur privilégié de transferts culturels. Quand un groupe d'individus se déplace, c'est un échantillon représentatif d'une nation qui se déporte d'un espace à un autre. Il y a forcément une reproduction sociale et culturelle qui est assurée dans ce nouvel espace par la poignée de personnes déplacées, aussi petite soit-elle. Autant il y a eu migrations et transferts culturels de l'Europe à l'Amérique, autant on peut observer ce même phénomène du côté de l'Afrique vers l'Europe. Le Burkina-Faso, ancienne colonie française, compte plusieurs milliers de ses fils dans l'Hexagone. Ces derniers ont emporté avec eux des traditions, us et coutumes de leur pays. Consciemment ou inconsciemment, en terrain favorable ou hostile, ils reproduisent ces pratiques. Parmi elles, on peut citer la solidarité, la polygamie, le partage, le regroupement... Dans ce thème, nous allons démontrer que ce qui est valable en Amérique pour l'Europe l'est en Europe pour l'Afrique à travers l'exemple des Burkinabè vivant en France.
\end{abstract}

\section{Introduction}

Les cultures régionales, ethniques et même religieuses du BurkinaFaso transcendent les frontières nationales pour se retrouver dans d'autres contrées comme la France, par le truchement de la migration. Cette migration, vécue dans le contexte africain en deux temps (pendant et après la colonisation) et en deux modes (contraignant et volontaire), est un vecteur privilégié de transferts culturels, car elle est loin de sonner le glas de certaines expressions culturelles dans la société d'accueil. Les groupes des immigrés forment des minorités qui cherchent à se faire une place au milieu d'une culture nationale très forte. Si certains rejoignent le courant dominant, d'autres s'accrochent à leurs habitudes par leurs actions et leurs comportements, qui assurent la conservation de leur identité. Quand un groupe d'individus se déplace, c'est un échantillon représentatif d'une culture ou d'une communauté qui se déporte d'un espace à un autre. Certaines des valeurs et des habitudes de la société burkinabé sont peu influencées par la France technologique et moderne, car elles survivent et prospèrent du fait de la détermination des immigrés à les reproduire. La motivation qui guide les transferts et les pratiques des habitudes culturelles burkinabé en France est le sentiment de rester attaché au terroir et de tisser un système d'autodéfense symbolique face 
aux difficultés d'intégration. Nous tenterons de démontrer cet état des faits ici.

Le Burkina-Faso, ancienne colonie française, compte plusieurs milliers de ses fils dans l'Hexagone. Partis de force ou de gré, ces derniers ont emporté avec eux des traditions, us et coutumes de leur pays. Consciemment ou inconsciemment, en terrain favorable ou hostile, ils reproduisent certaines de ces pratiques. Parmi elles, on peut citer la solidarité, la parenté à plaisanterie, la gérontocratie, les totems, la langue, les habitudes culinaires, vestimentaires et autres. Nous ferons apparaître que ce qui est valable en Amérique pour les migrants d'Europe l'est pour les migrants d'Afrique en Europe, à travers l'exemple des Burkinabè vivant en France. Toutefois, précisons que nos informations concernent bien sûr les Africains, les Burkinabè de près et surtout les Moosé, ethnie majoritaire dont je connais mieux les traditions. La problématique est donc la suivante : comment s'observent les transferts culturels de l'Afrique vers l'Europe dans le cadre des migrations des Burkinabè en France? Nous retiendrons comme hypothèse de travail que, face à des difficultés d'intégration dans le nouvel espace qu'est l'Europe, les Africains développent un système d'autodéfense en reproduisant les traditions populaires, les habitudes et pratiques culturelles de leur milieu d'origine. Les articulations autour desquelles nous mènerons notre réflexion sont : l'état des migrations des Africains vers l'Europe : origine, manifestations, causes; les habitudes culturelles burkinabé reproduites en France; les habitudes culturelles non transférables, invisibles, mais déterminantes; les enseignements à tirer de l'examen de ce phénomène.

\section{I - L'état des migrations des Africains vers l'Europe : origine, manifestations, causes}

Le phénomène des migrations est complexe, multiforme, car il constitue un facteur non négligeable de restructuration des sociétés et de diversification des expressions culturelles. Il dépasse largement les dimensions politique et économique pour intégrer les facteurs socioculturels. Analyser les migrations des peuples africains vers l'Europe revient à rendre compte de la complexité des dynamiques culturelles qui découlent de cette mobilité et aussi à mesurer le phénomène des transferts culturels possibles. L'Afrique se caractérise par une diversité de peuples et le déplacement permanent de ces derniers est un fait historique et social dont l'origine est déjà vieille d'un siècle.

La problématique des migrations est intimement liée au phénomène colonial qui a facilité un transfert presque forcé des bras valides vers l'Occident. Beaucoup d'économies occidentales ont pu se remettre sur pied par ce biais. Par exemple, la reconstruction de l'Europe depuis 
1945 s'est faite grâce à un appel de main-d'œuvre d'origine étrangère, majoritairement africaine. Le maintien des relations politiques, économiques, sociales et culturelles avec l'ancienne puissance coloniale au lendemain des indépendances a accéléré un déplacement massif de populations africaines vers l'Europe. Toutes les régions africaines (Ouest, Centre, Nord, etc.) connaissent cet exode de leurs populations vers plusieurs destinations en Europe, même si le Maghreb et l'Afrique de l'Ouest sont assurément les régions qui comptent la plus forte concentration de migrants en France. Aussi, les facteurs culturels, politiques, religieux, économiques sont les formes sous lesquelles il se manifeste.

La migration des Burkinabè vers la France a des causes indéniables et constitue une expérience inédite d'observation du syncrétisme culturel, qui mérite une attention particulière. Ces causes sont similaires pour tous les pays africains, même s'il faut d'emblée noter que le nombre des Burkinabè en France est minime par rapport à celui des autres nationalités. Seulement 5000 Burkinabè y vivent alors que le pays voisin, le Mali, dénombre 120000 de ses fils. Parmi les multiples causes, beaucoup de chercheurs en retiennent un certain nombre, que nous pouvons nommer classiques. Il s'agit des causes historiques qui trouvent dans la colonisation avec ses diverses formes d'exploitation (les travaux forcés, les gros travaux d'infrastructure, l'enrôlement sous le drapeau, l'institution des impôts) le facteur qui a engendré le goût des Burkinabè pour la vie à l'étranger. Les causes psychosociales sont aussi régulièrement citées et elles justifient l'immigration par l'imaginaire social : la recherche du prestige social et l'exhibition de fortunes acquises créent chez les jeunes gens une forte tentation de migrer. Enfin, les causes démographiques (la densité de la population qui pèse sur les terres inexploitées et appauvries) sont aussi importantes.

Au niveau des causes modernes, on peut citer les crises économiques, les crises culturelles et identitaires et les crises sociopolitiques.

\section{Les crises économiques}

Nul n'ignore que les économies africaines sont très fragiles, en raison de la faible mécanisation et de l'industrialisation balbutiante. Elles dépendent entièrement de l'Occident. La détérioration des termes de l'échange a contribué à compromettre ces économies, qui ont du mal à employer et à satisfaire les besoins des populations. C'est ainsi que ces dernières se déplacent vers l'Europe, à la recherche du mieux-être par la satisfaction des nécessités économiques (recherche d'argent pour satisfaire aux besoins domestiques et aux besoins sociaux). 


\section{Les crises culturelles et identitaires}

Le rôle de la politique coloniale dans les crises culturelles et identitaires fut déterminant. La conquête coloniale visait la soumission des citoyens à un nouvel ordre culturel. Jusqu'à la fin de la Seconde Guerre mondiale, la France a pratiqué dans ses colonies la politique d'assimilation, qui visait à faire de l'Africain un Français dans sa peau noire. Pour y parvenir, une transformation des institutions traditionnelles était nécessaire. Comme on le sait, quand deux cultures se rencontrent, la plus puissante phagocyte la plus faible, qui ne peut que perdre certains de ses repères. La modernité proposée par l'Europe à l'Afrique a hypothéqué la tradition africaine, en corrodant ses traditions, ses coutumes et certaines de ses valeurs. Cela pousse certains Africains à préférer rejoindre la modernité dans sa source.

\section{Les crises sociopolitiques}

Les maladies, la famine et les conflits interethniques sont devenus le lot de ce continent. La fragilité et la précarité des conditions de vie exposent les populations aux diverses maladies. Les caprices de Dame Nature rendent l'agriculture (activité qui occupe plus 60 pour cent de la population active) vivrière. L'autosuffisance alimentaire demeure un mirage pour bon nombre de pays africains, dont le Burkina-Faso. Les conflits surgissent d'un peu partout à cause de la mauvaise gestion des crises sociales et de la mauvaise manipulation de la diversité culturelle. La bonne gouvernance n'est pas au rendez-vous. Le Burkina-Faso, comme la plupart des pays africains, s'est engagé depuis plus de quinze ans dans la voie de l'édification d'une société démocratique pluraliste. Ce passage à la démocratie est jalonné de paradoxes et de contradictions. Les régimes autoritaires ont accéléré le départ vers l'Europe des intellectuels africains assoiffés de leur liberté et fiers de leurs opinions. Enfin, les études et la recherche constituent également une cause non négligeable de la migration. Les flux d'étudiants forment une catégorie en constante augmentation.

Peu ou prou, l'émigration vers la France constitue une réponse à la crise qui menace les sociétés villageoises. Ainsi, émigrer apparaît comme une sorte de promotion sociale qui accorde un statut social valorisant.

\section{II - Les transferts culturels : pratiques culturelles burkinabé reproduites en France}

La culture est considérée comme une sorte de "programmation mentale », c'est-à-dire que les modes de pensée, les sentiments, les valeurs, les symboliques et les systèmes de représentation sont le résultat d'un apprentissage continu. Les façons de penser, de sentir, 
d'agir s'installent dans l'esprit de chaque personne. On parle donc de "programmes mentaux ». Ainsi, l'horloger d'Europe centrale, avec son comportement poli toujours prisé, le garagiste américain qui s'est fait tout seul après une enfance misérable et l'Africain toujours au centre des soins de toute la communauté ont acquis des programmes mentaux différents. Autant d'environnements sociaux différents, autant de programmations mentales différentes ${ }^{1}$. L'étude des cultures révèle que les groupes humains pensent, sentent et agissent de façon différente et, dans un contexte de migration, le groupe d'immigrants laisse apparaître inconsciemment ses différences avec le peuple d'accueil. Les Burkinabè de France expriment leurs différences, preuves de leur programmation mentale, à travers des habitudes culturelles qui leur sont propres. Toujours selon Geert Hofstede, les différences culturelles se manifestent de plusieurs façons et quatre mots-clés permettent d'exprimer la totalité du concept de la culture. II s'agit des symboles, des rituels, des valeurs et des héros. Pour montrer le transfert des habitudes culturelles des Burkinabè de France, nous ferons appel tantôt aux valeurs tantôt aux symboles auxquels ces derniers se réfèrent, car eux seuls jouent un rôle primordial dans leur personnalité. Également, certains rites ou rituels, même s'ils sont difficilement transférables dans l'univers occidental, sont des repères importants que nous évoquerons. Deux préalables méritent d'être notés. Premièrement, les exemples de traditions, de coutumes que nous prendrons ne sont pas forcément et unanimement vécus par tous les Burkinabè de France dans leur totalité. Nos enquêtes ont touché des poignées d'immigrants (interrogés), qui reproduisent certaines pratiques culturelles que nous décrirons ici. Secondairement, certaines informations touchant à l'excision, au mariage polygamique et autres n'ont pas pu être vérifiées scientifiquement en raison de leur haute sensibilité et de leur grande délicatesse. Toute généralisation ou extrapolation systématique serait réductrice et conduirait à des stéréotypes ou à des préjugés qui handicaperaient les pistes sérieuses de réflexion.

Si les valeurs font partie des choses que les individus apprennent dès leur tendre enfance de façon inconsciente, mais méthodique et pédagogique dans le contexte africain, nous noterons que la solidarité, la gérontocratie, la parenté à plaisanterie et les interdits (alimentaires ou alliances) sont des valeurs cardinales pour les Burkinabè de l'intérieur et de l'extérieur.

1. Geert Hofstede, Vivre dans un monde multiculturel, Paris, les Éditions d'organisation, 1994, p. 8. 


\section{La solidarité}

Elle est une caractéristique légendaire du Burkina-Faso dans la mesure où elle a été enfouie dans l'imaginaire collectif par le truchement des enseignements des contes, des légendes, des initiations, des proverbes. Selon le principe d'Émile Durkheim ${ }^{2}$, il y a une différence entre les sociétés à solidarité mécanique, où la cohésion interne de la société découle de la force de la conscience collective du fait que les individus participent fortement au même sacré, et les sociétés à solidarité organique, dans lesquelles la cohésion sociale émane de la complémentarité des fonctions. Le passage des migrants burkinabé des sociétés traditionnelles de leur pays d'origine aux sociétés ultramodernes de l'Europe n'a pas entamé l'existence de cette forme de solidarité mécanique.

L'un des principes fondamentaux de cette solidarité est la conception particulière que les Burkinabè ont de la famille. Par exemple, les Moosé (ethnie majoritaire) possèdent une structure sociale solide, basée sur la famille. Il s'agit du buudu (communauté familiale). C'est à l'intérieur de ce cadre familial qu'est dispensée une éducation qui valorise le collectif. Le buudu regroupe la famille paternelle étendue, qui aide l'enfant à grandir et le protège. Le père et la mère en représentent le socle, auquel l'enfant doit respect et obéissance. La vieille Afrique dit : "L'homme d'abord! » La raison en est qu'il faut, selon cette Afrique, s'attacher à connaître l'autre, car il est riche d'observations et d'études pertinentes. Voilà pourquoi on éduque à la fraternisation. Cette façon de voir s'étend de la famille à la communauté et, dans le cas d'une migration, jusqu'à l'appartenance à la nation burkinabé et même, curieusement, au continent. Dans le contexte européen, elle se développe dans le réseau des associations. Ces dernières prennent la place des communautés villageoises des pays d'origine. Elles sont les cadres dans lesquels s'expriment les liens de vie commune et l'attachement à des valeurs partagées. Les Burkinabè de France comptent plus de douze associations. Ces regroupements correspondent souvent à des réalités provinciales ou départementales. Ce sont les ressortissants d'une même zone ou d'une même ethnie qui se retrouvent pour rechercher ou garder une cohésion sociale, culturelle et économique. En clair, cette solidarité a deux expressions.

La cohésion culturelle se traduit par la poursuite du partage des mêmes convictions et des mêmes sacrés. Les rencontres sont des occasions de revivre les traditions et les pratiques fondamentales de la zone d'origine. Par exemple, la zone peut être une réalité ethnique et, dans ce cas, l'un des objectifs de la structure peut être la promotion et la sauvegarde des traditions et habitudes de cette ethnie. C'est

2. Émile Durkheim, De la division du travail social, Paris, Alcon, 1893, p. 19. 
ainsi que, tous les ans, l'association Adabias (l'Association d'amitié burkinabé interculturelle autour du Sanguié) organise dans les locaux de l'ambassade du Burkina-Faso, à Paris, une exposition-vente de produits artisanaux et une dégustation des mets burkinabé et surtout gourounsi (ethnie du Burkina-Faso). Cette association signifie en langue liélé, en pays gourounsi, « les enfants d'un même père ». Elle a été créée en 1998 par le père Henri Leroy, qui a servi dans la paroisse de Réo. Son objectif est de cultiver l'entraide entre les ressortissants de cette province : "Le but d'ADABIAS est d'abord de nous réunir, de cultiver l'esprit de solidarité entre les membres, toutes générations confondues, et de transmettre aux jeunes les valeurs de fraternité et le sens de la famille. Nous faisons nôtre ce proverbe qui dit que "tout homme est un remède pour son semblable" ", nous explique la présidente de l'association, Sabine Kantiono. Avec ses activités, cette association sert de vitrine pour la découverte de la culture gourounsi.

L'expression économique : avec les fonds (les cotisations de ses membres, les dons et les recettes engendrées par la vente des produits artisanaux) récoltés, l'association aide plusieurs personnes avec des prêts sans intérêt. Également, cette association prend en charge les frais de scolarité des écoliers depuis l'école primaire jusqu'au lycée.

Le deuxième exemple est donné par les Burkinabè de Brest, dans le Finistère. En effet, chaque année la communauté de la cinquantaine des Burkinabè vivant à Brest organise une fête culturelle. Elle sert de vitrine de connaissance et de partage du patrimoine culturel, musical, et culinaire de ce pays. En 2007, elle s'est tenue à Treavergate (ville située à 16 kilomètres de Brest) avec la collaboration du foyer rural et de la municipalité de cette ville. Les recettes permettront de financer les projets de développement au Burkina-Faso. Ces Burkinabè se rencontrent, échangent et partagent ensemble leurs joies et leurs peines.

\section{La gérontocratie}

Dans la société traditionnelle burkinabé, le principe de la gérontocratie guide fondamentalement les actes d'éducation et d'initiation. Ce principe place le respect scrupuleux des aînés au centre du bon fonctionnement de la communauté. Partant de la logique que l'aîné est pétri d'expériences de la vie et habitué aux rouages ou mécanismes de pacification des relations humaines et de résolution des problèmes, la communauté le désigne comme un potentiel garant des us et coutumes. Manquer de respect à ce dernier est un signe visible du dénigrement futur des valeurs traditionnelles et culturelles. Dans l'observation des modes de vie des migrants, on remarque cette observance du respect envers l'aîné, car généralement c'est chez lui que les rencontres se tiennent. II reste la 
pierre angulaire dans la résolution des divers problèmes que rencontre chaque immigré. Il est un fin rassembleur et un parent pour eux tous. L'aîné des Burkinabè de Brest, par exemple, est au centre de toutes les initiatives : celles tendant à faire connaître le pays des hommes intègres et celles recherchant des solutions aux différents problèmes que les uns et les autres rencontrent en matière d'hébergement et d'emploi. Tout est fait comme si le plus âgé installé en France reçoit de la communauté le pouvoir d'être le chef de famille à qui on doit obéissance et respect, mais qui doit, en contrepartie, apporter secours à chacun et être un recours pour tous. C'est par ce biais que l'ordre villageois et la hiérarchie continuent à régner à des milliers de kilomètres.

\section{La parenté à plaisanterie}

Il s'agit d'une pratique sociale qui renforce la cohésion nationale, étant entendu qu'elle réduit de façon significative toutes les velléités de compétitions et de rivalités interethniques qui font des victimes dans certaines contrées. La parenté à plaisanterie désigne des taquineries diverses et des licences verbales échangées entre deux personnes d'ethnies différentes, dont l'objectif est la recherche de l'harmonie entre les groupes potentiellement rivaux. En clair, retenons que les alliances et les parentés à plaisanterie sont des formes de communication sociale entre des parents d'histoire commune. Elles font partie des différents garde-fous que s'était donnée l'Afrique pour préserver la paix et favoriser la coexistence pacifique. Le principe est de simuler le conflit pour éviter de rentrer réellement en désaccord avec l'autre. L'humour et l'ironie sont les figures utilisées pour taquiner le parent ou l'allié. Les Burkinabè vivant en France n'ont pas laissé disparaître cette pratique sur leur terre d'accueil. Ils la perpétuent en la pratiquant entre eux. Quand les parents ou alliés, dont les procédés et les traditions l'autorisent, se retrouvent, ils s'insultent et se moquent les uns des autres comme s'ils s'étaient au Burkina-Faso. C'est un réflexe traditionnel qui survit.

\section{Les totems}

La vie des Burkinabè en France ne se déroule pas en marge des totems auxquels les différents individus sont astreints. Les totems peuvent s'exprimer sous la forme des interdits alimentaires ou des interdits d'alliances conjugales. Au Burkina-Faso, chaque ethnie ou chaque village ou même, très souvent, chaque famille a ses interdits (totems). Les interdits alimentaires comme la proscription de la consommation de viande de certains animaux (cheval, chien, âne, lion, etc.) ou de certaines espèces végétales, de l'acte de tuer certaines espèces animales ou certains reptiles (comme le serpent) trouvent leur fondement dans les 
mythes et légendes fondateurs des villages ou des ethnies concernés. De même, la prohibition des alliances entre certaines castes est souvent de rigueur dans les comportements des immigrants burkinabé. Par exemple, les Yarsé ne peuvent pas contracter un mariage avec les Peulhs ni avec les forgerons. Nos enquêtes auprès des Burkinabè de France révèlent un respect scrupuleux de ces interdits dans l'Hexagone. A contrario, les mariages arrangés, orchestrés depuis le pays d'origine par les parents de l'immigré, sont pratiqués et servent de terreau de conservation des habitudes culturelles. La preuve est que le foyer composé de femmes et d'hommes burkinabé est l'endroit idéal où on mange, s'habille, parle et donc vit à la burkinabé.

Ensuite, il est évident que les symboles renvoient aux dessins ou aux objets porteurs d'une signification particulière, identifiables uniquement par ceux qui partagent cette culture. L'alimentaire, le vêtement, la coiffure, le langage ou le jargon, les rythmes musicaux et les spectacles appartiennent à cette catégorie des expressions culturelles.

\section{Les habitudes alimentaires}

La prise des repas est presque un moment solennel chez les peuples d'Afrique et plus particulièrement chez les Burkinabè. Elle réunit tous les protagonistes, c'est-à-dire non seulement les hommes et les jeunes qui se retrouvent à ce moment autour du plat, mais surtout les esprits et les animaux de la brousse. Le repas est en lui-même un rite sacré ("le plat est roi »). Il est véritablement le symbole par excellence de "la communion universelle entre vivants et morts ", "qui ne sont pas morts "3. Les repas sont collectivement pris au milieu des cours ou des champs. Les mets sont généralement tirés des denrées locales. Si en France, les Burkinabè ne peuvent pas prendre quotidiennement les repas ensemble, ils profitent des occasions que sont les mariages et les baptêmes pour le faire. Par ailleurs, ils retrouvent facilement les mets de leur pays grâce aux nombreuses boutiques de légumes et denrées africaines qui existent un peu partout. Nous avons remarqué l'existence de ces boutiques à Brest et à Neuilly. Elles sont des lieux d'approvisionnement des produits constitutifs des mets proprement africains. On peut citer les ingrédients comme le soumballa, la pâte d'arachide, les variétés de feuilles de sauge, les poissons fumés, etc.

3. Birago Diop, Les contes d'Amadou Koumba, par Bernard Mouralis, Paris, Bertrand Lacoste, 1991, p. 49. 


\section{L'art vestimentaire et capillaire}

Chaque peuple a ses habitudes propres pour l'habillement et la coiffure. L'art vestimentaire et capillaire constitue le trait le plus visible de notre appartenance à un groupe humain. Malgré les contraintes climatiques, l'art vestimentaire et capillaire burkinabé survit en France et connaît même des adeptes au sein de la population française. Les habits en cotonnade et toutes les parures propres au Burkina-Faso font l'objet de points de vente en plein Paris et dans les régions françaises. L'utilisation des nappes de table en batik, le port des habits traditionnels de style Dagara et Moosé ont été observés dans beaucoup de foyers.

\section{La langue}

La langue est un système de codes et de signes qui permet à une communauté large ou limitée d'échanger des messages. Pour Adama Samassékou, le président de l'Académie africaine des langues, "[d]e tous les éléments qui caractérisent l'individu, du physique au vestimentaire, c'est la langue qui demeure l'élément le plus évident [...] »4 . Les langues sont de véritables véhicules de cultures et de valeurs morales des sociétés africaines. Au Burkina, autant d'ethnies, autant de langues. Les Burkinabè, une fois en France, utilisent cet atout linguistique pour communiquer entre eux, soit à l'intérieur d'une même ethnie soit dans un cercle beaucoup plus large qui intègre d'autres personnes d'ethnies différentes. La langue locale aide les émigrés très souvent à s'évader dans un univers étranger au monde occidental pour se dire des secrets et des indiscrétions.

\section{Les rythmes musicaux et les spectacles}

Le Burkina-Faso, carrefour culturel de l'Afrique de l'Ouest, regorge d'artistes et de sonorités qui témoignent de la vivacité de ce secteur. Les artistes burkinabé, traditionnels comme modernes, égayent aussi les Burkinabè de l'extérieur, en se produisant sur leur demande à l'étranger afin de partager leurs productions artistiques avec eux. Les concerts et les spectacles du pays d'origine permettent aux immigrés de se ressourcer aux scènes et aux rythmes du terroir. Les Burkinabè de France reçoivent régulièrement les artistes du pays d'origine et préfèrent généralement les musiques venant de leur pays. Nous notons également les spectacles de contes et de théâtre des comédiens burkinabé qui drainent beaucoup de leurs compatriotes. De même, les expositions des photographes et des peintres permettent aux artistes burkinabé de communier avec

4. Adama Samassékou, «Avant-propos » du nºo du Bulletin ACALAN, Bamako, janvier 2002, p. 3. 
leurs frères. Par exemple, l'association Rivage, basée à Giou-de-Mamou, Auvergne, a organisé des activités culturelles du 2 au 30 juillet 2007, au cours desquelles il y a eu des expositions de photos, de dessins d'enfants et de tissages du Burkina-Faso. Les objectifs de cette association sont entre autres de faire connaître la culture des pays du Sahel, une bande de terre de 5,4 millions de $\mathrm{km}^{2}$ qui borde le Sahara et qui compte plus de 50 millions d'habitants. Les séances de contes du pays qu'elle organise ont bien permis aux jeunes Burkinabè qui connaissent très peu de choses sur leurs racines de découvrir cette vieille école de leur pays.

\section{III - Les habitudes culturelles non transférables, invisibles mais déterminantes dans la vie des immigrés burkinabé}

Les rituels ou rites sont des activités collectives ou individuelles, techniquement superflues pour parvenir au but désiré, mais considérées comme socialement essentielles dans une culture, car elles remplissent une fonction rituelle de recherche de bien-être. Dans la vie des Burkinabè de France, nous notons l'existence de certaines habitudes ou pratiques culturelles non transférables en raison du manque des conditions qui s'y prêtent. Les rites et les cultes aux ancêtres en sont des exemples. L'univers social des Moosé, par exemple, accorde une place prépondérante aux ancêtres, qui sont les garants des valeurs du groupe. Ce sont eux qui veillent sur la vie de la communauté. Le Moaga traditionnel redoute plus ses ancêtres que Dieu ${ }^{5}$. Le Burkinabè vivant à l'étranger a un lien fort avec ses défunts parents et ancêtres. Dans chaque grande famille, il y a des rites et cultes qui sont périodiquement exécutés dont le rôle est de prolonger le lien filial avec les morts. Ils s'expriment très souvent sous forme de sacrifices faits sur des symboles ou fétiches appartenant à la même lignée généalogique. Tout membre de la famille reconnaît son devoir ou son obligation de respecter ces pratiques s'il désire rester digne de cette communauté. Il est vrai que, avec la propagation des grandes religions, cette contrainte traditionnelle s'estompe progressivement, mais elle demeure observée par certains individus même vivant à l'étranger. C'est vrai qu'un Burkinabè vivant en France ne pourra pas faire des sacrifices sur les fétiches de son village qu'il ne peut pas faire venir par la poste. Néanmoins, il le fait par personne interposée.

Le recours aux forces invisibles fait partie de ces habitudes non transférables. Les Burkinabè ont une haute conception de leur milieu de vie (moogo) qu'ils considèrent comme le meilleur des mondes possibles. Le garant de ce meilleur des mondes est Dieu (Wendé) décliné en plusieurs

5. Alain Joseph Sissao, Contes du pays des Moosé, Paris, Éditions Karthala, 2002, p. 6. 
dimensions (Dieu des montagnes, celui des eaux et celui du bonheur). En clair, Dieu est assisté par les forces invisibles dont l'individu se sent beaucoup plus proche. De ce fait, le recours aux forces invisibles à travers la consultation des voyants et charlatans est une pratique courante. Très souvent, il ne procure qu'une satisfaction psychologique, car l'efficacité de ces forces ne résiste pas à la preuve scientifique. En effet, la rationalité africaine cherche une causalité du mal et du bien, du gain et de l'infortune dans l'action de l'homme. La pensée magique cherche une causalité en méprisant la réalité et les lois naturelles. Elle sert pour l'Afrique comme un axiome d'explication des événements de notre vie. On y recourt pour rechercher la réussite, la santé, la progéniture, en bref, le bonheur. Les immigrants burkinabé ne se dérobent pas de cette pratique et, par personne interposée dans le pays d'origine, ils consultent les spécialistes pour assurer leurs arrières. Il n'est pas exclu de voir des prétendus charlatans en plein Paris.

Mariage polygamique : la polygamie est une pratique courante dans notre pays. Seulement nos enquêtes nous ont permis de constater que les Burkinabè de France dans leur grande majorité ne la perpétuent pas. Cet état de fait trouve une partie de son explication dans la cherté de la vie d'une part et d'autre part dans les mariages mixtes (entre Burkinabè et Français). Du coup, la polygamie, qui est le fait qu'un seul homme épouse et vive maritalement dans une même concession avec deux ou plusieurs femmes, n'est pas reproductible en France. En revanche, une autre forme de ce régime de mariage est observable chez certains. Il s'agit d'avoir une femme dans le pays d'origine et d'en épouser une autre en France. Très souvent chacune ignore l'existence de l'autre.

Le phénomène de l'excision : il s'agit d'un vieux fléau qui traumatise la gent féminine burkinabé. Les multiples stratégies de sensibilisation aux méfaits de cette pratique n'arrivent pas à mettre fin à cela. II arrive que, pour échapper aux sanctions pénales en vigueur sur le sol français, beaucoup de parents africains, adeptes de cette pratique ancestrale, renvoient leurs filles dans le pays d'origine afin qu'elles subissent ces rites initiatiques destinés à marquer leur entrée dans la catégorie des adultes.

\section{IV - Les enseignements à tirer de l'examen de ce phénomène}

\section{Le transfert des habitudes culturelles comme moyen de défense identitaire}

Dans l'échafaudage des chemins d'intégration, la reproduction des expressions culturelles locales et des habitudes culturelles est capitale puisque c'est sur elles que vont se greffer les autres possibilités 
d'adaptation. C'est le lieu d'invoquer la place de l'identité du point de vue interactionniste dans le processus migratoire. Il y a une reconstitution des symboles. Face aux diverses formes d'exclusion teintées culturellement, les immigrants expriment la diversité de leurs identités qui servent de rempart symbolique. La capacité d'intégration des sociétés, les prédispositions des acteurs de la migration conditionnent étroitement la question des identités. L'identité est une réalité sacrée, car c'est d'elle que les individus tirent la sève nourricière de leur présente existence. Elle est ce fondement sur lequel repose toute communauté composée des êtres désirant vivre ensemble. Les Burkinabè de France, en reproduisant certaines des expressions culturelles de leur société d'origine, se placent dans un réconfort qui leur procure une sorte de satisfaction morale, culturelle et traditionnelle dont ils ont besoin pour donner un véritable sens à leur vie et surmonter les difficultés. La migration, en soutirant les individus de leur espace d'origine, est vécue comme une sorte de déracinement. Elle entraîne une déculturation des individus. Or, tout comme un arbre déraciné est exposé aux intempéries naturelles, un homme déculturé est sujet à toutes les vicissitudes de son environnement de vie. Reproduire quelques bribes de sa culture dans son nouveau cadre de vie est une aubaine qui permet de pouvoir rester attaché à son terroir.

\section{Le transfert des habitudes à l'épreuve de l'ultra-modernisme}

Avec l'individualisme grandissant, nous remarquons que l'autodéfense identitaire par les transferts culturels est souvent handicapée par les préoccupations individuelles, qui rendent distants les rapports entre les migrants. Nés dans des sociétés dites « collectivistes » (sans connotation politique) où l'intérêt du groupe prime sur l'intérêt individuel, où le groupe d'appartenance est la principale source d'identité d'un individu, les immigrants, une fois dans ce nouvel espace, sont contraints d'adopter le principe selon lequel l'individuel prend le pas sur le collectif. Le côté collectiviste de leur comportement supplante le côté individualiste. Cet individualisme se manifeste par l'enfermement des hommes dans leurs familles modernes. Les enfants des familles nucléaires apprennent très vite le « je » qui est leur identité personnelle, distincte des autres $«$ je ${ }^{6}$. Les camarades sont choisis selon la préférence et l'éducation vise à apprendre à se débrouiller seul. Cela corrode les liens forts qui existent entre les immigrants et se manifestent par une solidarité mise à mal. Les contraintes et le rythme du travail favorisent également l'individualisme, car la profession en Europe rend le travailleur esclave du

6. Geert Hofstede, op. cit., p. 24 . 
temps. Il n'a pas assez de temps pour fréquenter ses compatriotes en vue de renforcer ses liens de fraternité, donc de solidarité.

\section{Conclusion}

La mondialisation a provoqué une crise identitaire et communautaire. Les communautés africaines sont aujourd'hui à la recherche de leurs identités particulières, car, dans ce monde en pleine mutation, les identités primaires ou originelles sont perdues ou en voie de l'être. Cette déperdition des valeurs est d'autant plus criarde dans le contexte de migration. C'est en ce moment que les initiatives et les stratégies doivent être trouvées par les autorités de notre pays pour qu'on puisse réellement faire de la culture non pas un simple legs spirituel de la tradition, mais un rempart symbolique derrière lequel chacun se réfugie pour défendre la signification de son existence. Les Burkinabè de France font de leur mieux pour conserver certaines de leurs habitudes culturelles, compte tenu du noble service qu'elles leur rendent dans le processus d'affirmation de leur appartenance à la société de base burkinabé. Il est important que les autorités burkinabé offrent des possibilités plus nombreuses à leurs compatriotes afin qu'ils puissent perpétuer, exprimer et partager leurs expressions culturelles dans l'Hexagone.

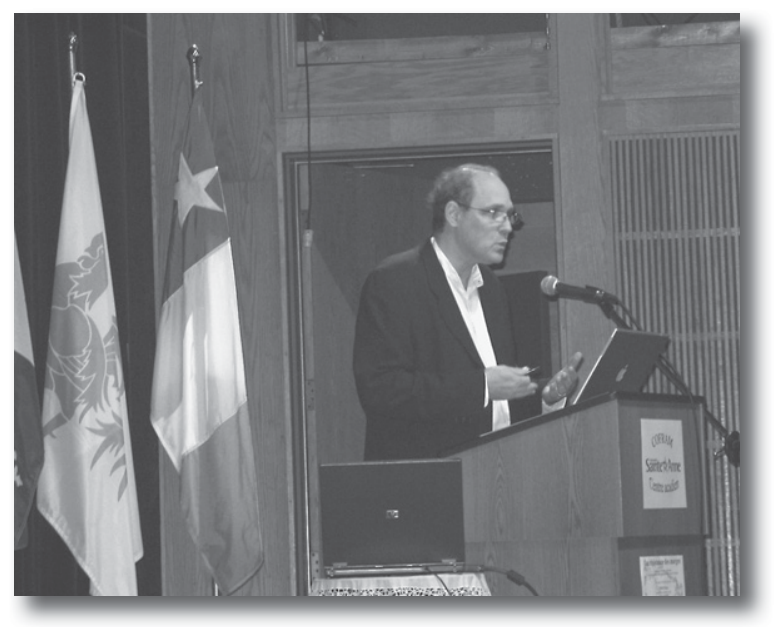

Yves Bergeron 